\title{
ON A POPULATION DYNAMIC MODEL OF ACTIVE CELLS WITH DIRECT INTERACTION
}

\author{
AHMEd Noussair
}

\begin{abstract}
Through two case studies, this paper deals with a new class of population dynamic models inspired from the kinetic theory for active particles modelling cell to cell interactions with a transfer processes between cells. The first case study problem is related to the transfer of proteins motivated by advantages of cell transfer therapies for the treatment of cancers. The second case concerns the activity transfer between immune and tumor cells. We provide some numerical tests and we prove the convergence of the solutions from the discrete model to the continuous model.
\end{abstract}

Mathematics Subject Classification. 35Q53, 34B20, 35G31.

Received October 30, 2017. Accepted November 13, 2017.

\section{INTRODUCTION}

The mathematical modeling of complex biological systems is one of the major challenges of this century. Complexity arises when large numbers of individuals (cells, bacteria, people) collectively organize into spatial patterns. The kinetic theory for active particles, hereafter in brief KTAP, has been developed in order to model complex systems constituted by a large number of interacting particles (called active particles), whose microscopic state includes, in addition to position and velocity variables, also a scalar variable (called activity variable), which is related to the intrinsic biological/social function of particles. The interested reader on recent developments of this theory is referred to the bibliography section of the books [3-5].

The subject of this paper is the modeling of transfer processes occurring between population of cells in a living system constituted by a large number of entities which interact with different strategies and exchange some quantities of transferable materials or exchanging some inhibition or activation of activities due to some signal transfers. In this paper, we consider two cases of study. The first problem is related to the transfer of proteins or a transferable matter between interacting cells. The work is motivated by advantages of cell transfer therapies for the treatment of cancers $[8,9]$, while the second problem concerns transfer of activity between immune and tumor cells. The motivation is that the stage of the early growth of a tumor belongs to the socalled free cell regime, in which the tumor cells are not yet condensed in a macroscopically observable spatial structure, and the interaction between tumor and immune system occur at the cellular level. This makes the kinetic approach particularly appropriate. The goal is to provide some new models for transfers which can be used in practice to fit real experimental data.

For both case studies presented here, the mathematical framework is defined by a system of integrodifferential equations which describe the evolution in time of the distribution function over the state of cells at the cell level.

Keywords and phrases: Cell-to-cell interaction, structured population dynamic, Boltzmann like-type models, kinetic theory of active particles, numerical analysis.

UMR CNRS 5251 IMB, Université de Bordeaux, Bordeaux, France. Ahmed.noussair@u-bordeaux.fr 
This makes the kinetic approach particularly appropriate. The situation can be regarded as particular cases of methods of mathematical kinetic theory for active particles. This theory is a part of a general mathematical framework of the "Theory of Active Particles" introduced by Bellomo [3]. It can be regarded as new mathematical approach which develops method of kinetic theory to deal with active particles (cells) rather than classical particles. The internal state of cells includes biological functions. The modelling of microscopic interactions also refers to the ability and the behavior of cells to interact and communicate with other cells, including proliferation and destruction $[3-5]$.

An interesting prospect is that biological transfer processes will be supported by rigorous investigation methods and tools, similar to what happened in the case of mechanical and physical sciences. It is not an easy task, considering that new mathematical methods may be needed to deal with the inner complexity of biological systems which exhibit features and behaviors very different from those of inert matter. Indeed, cells organize their dynamics according to the above functions, while classical particles follow deterministic laws of Newtonian mechanics.

For more information on biological transfers processes, recent studies have shown that cells can communicate by the transfer of membrane proteins [7]. Inter-cellular transfer of proteins is a mode of communication between cells that is crucial for certain physiological processes. For instance, the direct transfer of protein P-glycoprotein (P-gp) between cells was studied in $[1,11]$. Because P-gp may act as a drug-efflux pump, its transfer may confer resistance against cytotoxic drugs to cancer cells. Another example is the transfer of human immunodeficiency virus from an infected cell to an uninfected cell [6]. It has been shown in recent studies that the $\alpha$-synuclein can transfer from one cell to another and could be a key element in the spread of Parkinson's disease pathology [10].

The paper is organized as follows: in Section 2, we formulate the general model of population dynamic governing cell to cell interaction. In Section 3, through the microscopic interactions of proteins transfers problem, we describe the transfer rules, we then derive a fully discrete model and we give its associated continuous model and prove the convergence from discrete model to the continuous model is postponed at the Appendix B. In Section 4, we consider two population of cells, immune cells and tumor cells, exchanging their level of activities, we formulate a continuous nonlinear model and we give a numerical scheme and a priori estimates needed to prove the convergence. In the next section we present some numerical results.

\section{A GLOBAL FORMALISM OF A POPULATION DYNAMIC MODEL}

In this section we introduce a new class of population dynamics model. This class of models can be seen as a particular case of models of kinetic theory of active particles without mechanical and spatial structure. The advantage of these models is to focus on the biological phenomenon of transfers processes between interacting cells and to avoid the mathematical complexity to deal with spatial structure and Boltzmann equations which are not easy to study mathematically. Continuous structured population models consider a population described by a density $u(x, t)$ of individuals with respect to a variable $x$, sometimes called internal, structuring, or physiological variable, with $x$ in $\left[x_{0}, L\right), L>0$, and $x_{0}>0$. Examples of these variables are age, size, height, body surface, energy reserve or maturity. In their classical form, these models are formulated in terms of nonlocal first-order hyperbolic PDE (a balance law) governing the dynamics of the density $u(x, t)$.

In order to derive the model we need to introduce some assumptions:

Assumption (A1). The physical system is constituted by $n$ interacting cell populations. Each population individual may be found in a state described by a variable $x \in[0,1]$.

Assumption (A2). The probability density functions is defined, for each population, by

$$
u^{k}=u^{k}(t, x):[0, \infty) \times[0,1] \longrightarrow \mathbf{R}^{+} .
$$

Therefore, the probability of finding, at the time $t$ an individual of the $i$-population in the state interval $[0,1]$ is given by

$$
P_{i}(t)=\int_{0}^{1} u^{k}(t, x) \mathrm{d} x
$$


Assumption (A3). Interactions can be subdivided into conservative encounters which modify the state of the cells but not their number. Cell interactions will be defined by means of the two quantities: the encounter rate $\eta^{k l}$ and the transition probability density $\psi^{k l}$. The rate $\eta^{k l}(y, z)$ denotes the number of encounters per unit volume and unit time between cell pairs of the $(k, l)$ th populations with states $y$ and $z$, respectively, we have

$$
\eta^{k l}(y, z)=\eta^{k l}(z, y)
$$

and $\psi^{k l}(y, z, x)$ denotes the probability of transition of the $k$ th cell to the state $x$, given its initial state $y$ and the state $z$ of the encountering cells belonging to the $l$ th population.

Assumption (A4). Proliferating cells will be described by two processes. The first process is due to the proliferation with the smallest size value of $x$ (here assumed to be $x_{0}=0$ ). The number of new cells are

$$
\int_{0}^{1} \beta_{1}^{k}(P(t), x) u^{k}(t, x) \mathrm{d} x
$$

The vital birth rate $\beta^{k}(\cdot, x)$ denotes the number of cells produced per unit volume and unit time of the $(k)$ th species with state $x$. While the second process concerns the proliferation with distributed sizes values of $x$.

$$
\int_{0}^{1} B^{k}(P(t), x, \xi) u^{k}(t, \xi) \mathrm{d} \xi
$$

Different newborns can have different values of $x$, for instance, cells with different sizes split into two new cells, there is not a fixed size for daughter cells coming in the cell population. The nonlinearities are defined by the vector of variables

$$
P(t)=\left(P_{1}(t), \ldots, P_{n}(t)\right)^{T}
$$

Assumption (A5). Destructive cells will be described by the vital death rate $\mu^{k}(P(t), x)$ which is the number of $k$ th species with state $x$ destroyed.

The evolution equation obtained using the above assumption consists of the following system of $n$ coupled integro-differential equations:

$$
\left\{\begin{array}{l}
\frac{\partial u^{k}(t, x)}{\partial t}+\underbrace{\frac{\partial\left(v^{k}(P(t), x) u^{k}(t, x)\right)}{\partial x}}_{\text {Self activity-growth }}+\underbrace{\mu^{k}(P(t), x) u^{k}(t, x)}_{\text {Apoptosis }} \\
=\underbrace{\sum_{j=1}^{n} Q^{k l}\left(u^{k}, u^{l}\right)(t, x)}_{\text {Transfer }}+\underbrace{\int_{0}^{1} B^{k}(P(t), x, \xi) u^{k}(t, \xi) \mathrm{d} \xi}_{\text {Distributed proliferation }}, \\
v^{k}(P(t), 0) u^{k}(t, 0)=\underbrace{\int_{0}^{1} \beta^{k}(P(t), x) u^{k}(t, x) \mathrm{d} x}_{\text {Punctual proliferation }}, \\
u^{k}(t, 0)=u_{k, 0}(x),
\end{array}\right.
$$


where

$$
Q^{k l}\left(u^{k}, u^{l}\right)=Q^{k l+}\left(u^{k}, u^{l}\right)-Q^{k l-}\left(u^{k}, u^{l}\right),
$$

with

$$
\begin{aligned}
& Q^{k l+}\left(u^{k}, u^{l}\right)(t, x)=\int_{0}^{1} \int_{0}^{1} \eta^{k l}(y, z) \psi^{k l}(y, z, x) u^{k}(t, y) u^{l}(t, z) \mathrm{d} y \mathrm{~d} z \\
& Q^{k l-}\left(u^{k}, u^{l}\right)(t, x)=u^{k}(t, x) \int_{0}^{1} \eta^{k l}(x, y) u^{l}(t, y) \mathrm{d} y .
\end{aligned}
$$

$Q^{k l+}$ and $Q^{k l-}$ correspond, respectively, to the gain and loss of cells in the state $x$ due to conservative encounters.

Remark. In order to have a well-posed initial value problem (IVP) one needs an initial condition $u_{0}(x)$ and a boundary condition at $x=x_{0}$. Usually, this boundary condition describes the inflow of newborns in the population, i.e. the inflow of individuals having a value $x=x_{0}>0$ of the structuring variable. It is likely that this sort of boundary condition comes from the fact that the first structuring variable considered in the literature was age and, in this case, it is clear that all the newborns have exactly the same value of age-at-birth, namely $x_{0}=0$. For instance, if $x_{0}$ denotes the size-at-birth of an individual, different newborns can have different values of $x_{0}$, for instance, in cell populations where large enough cells with different sizes split into two new cells, there is not a fixed size for daughter cells coming in the cell population.

\section{First CASe Study: Direct CELL to CELL PROteins transfers}

\subsection{Transfer rules}

Consider a population of cells in a co-culture, where each cell possesses an amount of protein with size $x$, in $\left[x_{\min }, x_{\max }\right]$ to be partially transferred due to some specific rules. Assume that cells continually encounter other cells. Each pairwise of encounter during the transfer time results in a winner "Recipient cell" and a loser "Donor cell" or a loser "Recipient cell" and a winner "Donor cell". Then in order to fully determine the transfer rules we will use two types of (deterministic type) transfers (Fig. 1).

The transfer rules read as follows:

Let $x_{j}^{\text {old }}, x_{k}^{\text {old }}$ are the pre-transfer content sizes before transfer of the interacting cells, and whose difference in quantity is $l=x_{k}^{\text {old }}-x_{j}^{\text {old }}$, and let $x_{j}^{\text {new }}, x_{k}^{\text {new }}$ are the post-transfer content sizes, that are related to $x_{j}^{\text {old }}$ and $x_{k}^{o l d}$ by the relations

1. First transfer with transfer efficiency $f_{1}$ "Recipient cells win"

$$
x_{j}^{\text {new }}=x_{j}^{\text {old }}+f_{1}(l) l, \quad x_{k}^{\text {new }}=x_{k}^{\text {old }}-f_{1}(l) l .
$$

In this situation, the size of the content increases from $x_{j}^{\text {old }}$ to a value $x$ then $y_{j}^{\text {new }}=x$ and $x_{j}^{\text {old }}=x-f_{1}(l) l$. 2. Second transfer with transfer efficiency $f_{2}$ "Donor cells win"

$$
x_{j}^{\text {new }}=x_{j}^{\text {old }}+f_{2}(l) l, \quad x_{k}^{\text {new }}=x_{k}^{\text {old }}-f_{2}(l) l .
$$

In this situation, the size of the content is decremented from $x_{k}^{\text {old }}$ to a value $x$ then $x_{k}^{\text {new }}=x$ and $x_{k}^{\text {old }}=x+f_{2}(l) l$.

The probability $\pi_{1}(l)$ that the "Recipient cells win" and the probability $\pi_{2}(l)$ that the "Donor cells win". Because of the two events "Recipient wins, Donor loses" and "Recipient loses, Donor wins" are 


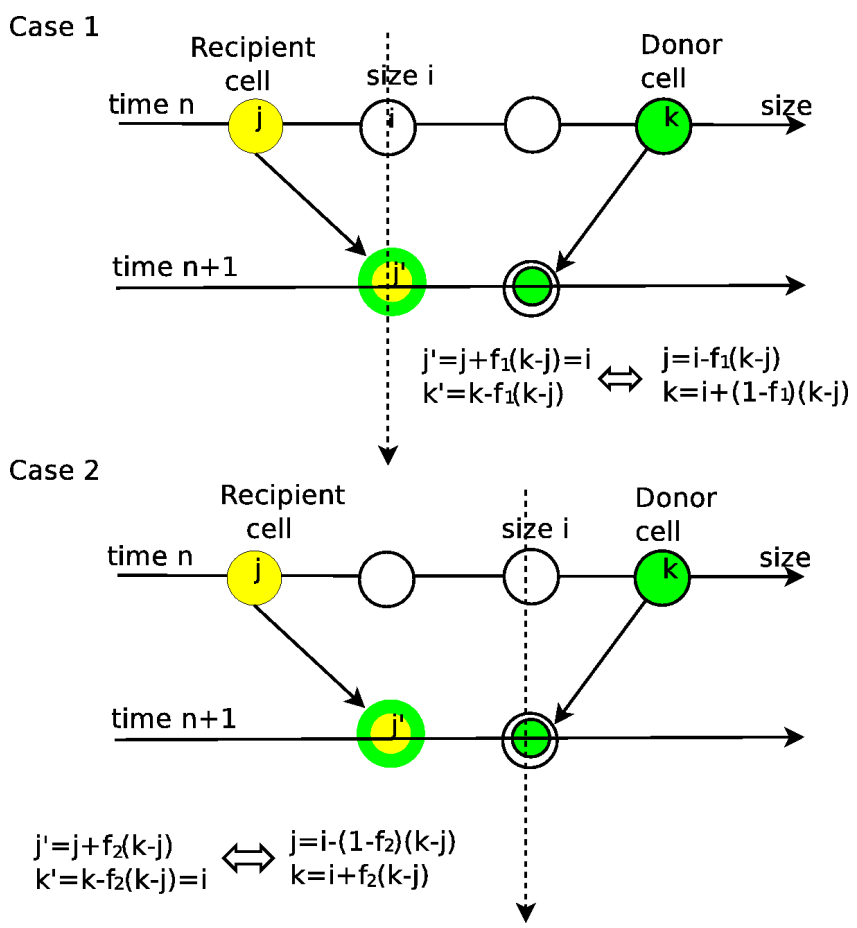

Figure 1. Cell to cell direct transfer rules.

complementary we have

$$
\pi_{1}(l)+\pi_{2}(l)=1 .
$$

The first type of transfer will occur with an efficiency rate function $f_{1}(\cdot)$ and with a probability $\pi_{1}(\cdot)$. The second type of transfer will occur with efficiency function $f_{2}(\cdot)$ with a probability $\pi_{2}(\cdot)$. The basic assumptions used to describe the transfers are the following.

\subsubsection{The discrete model}

Given the maximal value of the transferable quantity $x_{\max }$ and its minimal value $x_{\min }$, we consider a partition

$$
x_{\text {min }}=x_{1}<x_{2}<\cdots<x_{i}=x_{1}+(i-1) \Delta x<\cdots<x_{I_{L}}=L,
$$

with $x_{i+1}-x_{i}=\Delta x, \forall i=1, I_{L-1}$. Without loss of generality, for simplicity we assume that $x_{\min }=x_{1}=0$ and $x_{\max }=x_{I_{L}}=L=1$. Starting from an initial distribution $u_{0}(x)$ for all sizes $x$ in $[0,1]$, we introduce an initial sequence $u_{i}^{0}, i=1, \ldots, I_{L}$, by

$$
u_{i}^{0}=\frac{1}{\Delta x} \int_{x_{i}}^{x_{i+1}} u_{0}(x) \mathrm{d} x \simeq u_{0}\left(\frac{x_{i+1}+x_{i}}{2}\right)
$$

We suppose that all the values $u_{i}^{n}$ for $i=1, \ldots, I_{L}$ are known, and we propose to build the $u_{i}^{n+1}$ for $i=1, \ldots, I_{L}$ by the following scheme: 


$$
\begin{aligned}
u_{i}^{n+1}= & u_{i}^{n}+\Delta t \sum_{j} \tilde{\eta}_{i j}^{1} \pi_{1}\left(l_{j}\right) \underbrace{\bar{u}_{i-f_{1}\left(l_{j}\right) l_{j}}^{n} \Delta x}_{\text {Recipient cells }} \underbrace{\bar{u}_{i+\left(1-f_{1}\left(l_{j}\right)\right) l_{j} \Delta x}^{n} \Delta x}_{\text {Donor cells }} \\
& +\Delta t \sum_{j} \tilde{\eta}_{i j}^{2} \pi_{2}\left(l_{j}\right) \underbrace{\bar{u}_{i-\left(1-f_{2}\left(l_{j}\right)\right) l_{j} \Delta x}^{\Delta}}_{\text {Recipient cells }} \underbrace{\bar{u}_{i+f_{2}\left(l_{j}\right) l_{j} \Delta x}^{n}}_{\text {Donor cells }} \\
& -\Delta t \underbrace{u_{i}^{n} \sum_{j} \eta_{i j} u_{j}^{n}}_{\text {To other sizes }}+\Delta t \underbrace{\sum_{j} B_{i, j}^{n} u_{j}^{n} \Delta x}_{\text {Proliferation }},
\end{aligned}
$$

where $l_{j}=j \Delta x$ is the difference of the transferable quantities between two partner cells, the quantities $i-$ $f_{1}\left(l_{j}\right) l_{j}, i+\left(1-f_{1}\left(l_{j}\right) l_{j}, i-f_{2}\left(l_{j}\right) l_{j}\right.$ and $i+\left(1-f_{2}\left(l_{j}\right) l_{j}\right.$ are understood respectively as their integer part in the system (3.1), and

$$
\bar{u}_{i}:= \begin{cases}u_{i} & \text { if } i=1, \ldots, I_{L} \\ 0 & \text { otherwise }\end{cases}
$$

where $\tilde{\eta}_{i j}^{1}=\eta\left(x_{i-f_{1}\left(l_{j}\right) l_{j}}, x_{i+\left(1-f_{1}\left(l_{j}\right)\right) l_{j}}\right), \tilde{\eta}_{i j}^{2}=\eta\left(x_{i-f_{2}\left(l_{j}\right) l_{j}}, x_{i+\left(1-f_{2}\left(l_{j}\right)\right) l_{j}}\right)$ and $\eta_{i j}=\eta\left(x_{i}, x_{j}\right)$, with $\eta(x, y)$ is the encounter rate between cells with size $x$ and cells with size $y$. Let us rewrite the above scheme under the compact form

$$
u_{i}^{n+1}=u_{i}^{n}+\Delta t Q_{i}^{n}+\Delta t \sum_{j} B_{i, j}^{n} u_{j}^{n} \Delta x
$$

with

$$
Q_{i}^{n}:=\sum_{j} \sum_{k} \psi_{i j k} \eta_{i j} u_{j}^{n}(\Delta x)^{2} u_{k}^{n}-u_{i}^{n} \sum_{j=1} \eta_{i j} u_{j}^{n} \Delta x
$$

with

$$
\psi_{i j k}=\pi_{1}\left(x_{k}-x_{j}\right) \delta_{i, j+f_{1}\left(x_{k}-x_{j}\right)}+\pi_{2}\left(x_{k}-x_{j}\right) \delta_{i, k-\left(1-f_{2}\right)\left(x_{k}-x_{j}\right)},
$$

where $\delta_{i, j}$ is the Kronecker symbol. For instance, in cell populations where large enough cells with different sizes split into two new cells, there is not a fixed size for daughter cells coming in the cell population. For an equal cell division we have

$$
B_{i, j}=4 b_{i} \delta_{2 * i, j}-b_{i} \delta_{i, j}
$$

\subsubsection{From discrete to continuous model}

In this section we show the convergence of the scheme (3.1). It means that the difference $\left|u-u^{\Delta}\right|_{1}$ between the approximate solution $u^{\Delta}$ of the discrete model (3.1) and the solution $u$ of the continuous model (5.2) tends to zero as the mesh size $\Delta t, \Delta x$ goes to zero (for simplicity we set $\Delta t=r \Delta x$ ). Here the approximate solution 
$u^{\Delta}$ is defined as the piecewise constant function defined on $] 0, T[\times] 0,1[$

$$
\left.u^{\Delta}(t, x)=u_{i}^{n} \quad \text { for all }(t, x) \in\right] t_{n}, t_{n+1}[\times] x_{i}, x_{i+1}[,
$$

where the values $u_{i}^{n}$ are computed by (3.1) and $u$ is the solution of the following continuous Cauchy problem:

$$
\left\{\begin{array}{l}
\frac{\partial u(t, x)}{\partial t}=Q(u(t, \cdot), u(t, \cdot))(x)+\int_{0}^{1} B(P(t), x, \xi) u(t, \xi) \mathrm{d} \xi \quad \text { for } x \in \mathbb{R} \\
u(0, \cdot)=u_{0} \in L_{+}^{1}(\mathbb{R}) .
\end{array}\right.
$$

The operator describing the rule for one transfer is defined by

$$
\begin{aligned}
Q(u, u)(x):= & \int_{\mathbb{R}} \eta(x, l) \pi_{1}(x) u\left(x+f_{1}(l) l\right) u\left(x-\left(1-f_{1}(l)\right) l\right) \mathrm{d} l \\
& +\int_{\mathbb{R}} \eta(x, l) \pi_{2}(x) u\left(x+f_{2}(l) l\right) u\left(x-\left(1-f_{2}(l)\right) l\right) \mathrm{d} l \\
& -u(t, x) \int_{\mathbb{R}} \eta(x, y) u(t, y) \mathrm{d} y .
\end{aligned}
$$

This can be rewritten as (2.2)-(2.4) with $\eta(y, z)=1$ and

$$
\psi(x, y, z)=\pi_{1}(x) \delta_{x-y-f_{1}(z-y)(z-y)}+\pi_{2}(x) \delta_{x-z+\left(1-f_{2}(z-y)\right)(z-y)} .
$$

And where the continuous process for an equal cell division is described by choosing $B(P(t), x, \xi)$ as follows:

$$
B^{k}(x, \xi)=4 b^{k}(2 x) \delta_{\xi-2 x}-b^{k}(x) \delta_{\xi-x}
$$

We have the following theorem holds:

Theorem 3.1. Let $u_{0} \in\left(L^{1} \cap L^{\infty}\right)(] 0,1[)$ with total variation bounded locally in $] 0,1\left[, u_{0} \geq 0\right.$, then as the mesh size $\Delta x$ tends to zero, there is a subsequence of $\left(u^{\Delta}\right)_{\Delta x>0}$, the family of approximate solution of the discrete model, converging in $L_{l o c}^{1}([0, T] \times] 0,1[)$ to a function $u \in L_{l o c}^{1}([0, T] \times] 0,1[)$ and the limiting function $u$ is solution of the solution of the continuous problem. Moreover, in the case with no proliferation, the first and second moment of the distribution $u_{0}$ are preserved in time. Namely we have the following properties for each $t \geq 0$ :

$$
\int_{\mathbb{R}} u(t, x) \mathrm{d} x=\int_{\mathbb{R}} u_{0}(x) \mathrm{d} x \text { and } \int_{\mathbb{R}} x u(t, x) \mathrm{d} x=\int_{\mathbb{R}} x u_{0}(x) \mathrm{d} x .
$$

Proof. See Appendices A and B.

\section{ACtivity TRANSFER IN TUMOR-IMMUNE SYSTEM INTERACTION}

\subsection{Assumptions}

In this case study, the framework proposed is specialized to model the immune competition at the cellular level between immune cells and abnormal cells. The immune system involves several different subpopulations of cells; however to simplify the complexity induced by considering a large number of subpopulations, we will consider, here, the immune cells as only one population. An analogous simplification will be carried out for the 
cells that are carries of a pathology (for instance cancer cells). Therefore we consider two families of cells which develop activities in their interactions and competition.

To precise the modelisation, the following specific assumptions will be needed:

Assumption (A1). The system is constituted by two interacting cell populations: environmental and immune cells, labelled, respectively, by the indexes $k=1$ and $k=2$ homogeneously distributed in space.

Assumption (A2). The functional state of each cell is described by a real variable $x \in[0,1]$. For the environmental cells, the above variable refers to the natural state (normal cells) for $x=0$ and to the abnormal state (abnormal cells) for $x \in] 0,1]$. For the immune cells, $x=0$ correspond to non-activity or inhibition; $x \in] 0,1]$ correspond to activation.

Assumption (A3). The encounter rate is assumed to be constant and equal to unity for all interacting pairs, hence $\eta^{k l}=\eta=1, \forall k, l=1,2$.

Assumption (A4). The transition probability density related to conservative interactions is assumed to be delta functions:

$$
\psi^{k l}(y, z, x)=\delta\left(x-m^{k l}(y, z)\right),
$$

where $m^{k l}$ corresponds to the output which may depend on the microscopic state of the interacting pair. The basic restrictions on $m^{k l}$ are their non-negativity, and also $0 \leq m^{k l}(y, z) \leq 1,0 \leq y, z \leq 1$, to ensure that dominance values range between zero and unity.

- Conservative encounters of abnormal cells: Cells of the first population show a tendency to degenerate with most probable output given as follows:

$$
m^{11}(y, z)=\left(1-\alpha^{11}\right) y+\alpha^{11}
$$

where $0 \leq \alpha^{11}<1$ is a parameter related to the inner tendency of both a normal and an abnormal cell to degenerate. Here, we consider that the cells do not show a natural tendency to degenerate, i.e. $\alpha^{11}=0$.

If an abnormal cell encounters an active immune cell, its state decreases with most probable output given as follows:

$$
m^{12}(y, z)=\left(1-\alpha^{12}\right) y,
$$

where $0 \leq \alpha^{12}<1$ is a parameter which indicates the ability of the immune system to reduce the state of cells of the first population.

- Conservative encounters of active immune cells: The only encounters with non-trivial output are those between active immune cells and abnormal cells:

$$
m^{21}(y, z)=\left(1-\alpha^{21}\right) y, \quad m^{22}(y, z)=y
$$

where $0 \leq \alpha_{21}<1$ is a parameter which indicates the ability of the abnormal cells to inhibit immune cells. For more detail see [5].

The last basic assumption in this model (that we will also take into account) is that the ingested energy is allocated between metabolic maintenance, individual growth and reproduction.

Assumption (A5). The structuring variable is the level of the activity of cells. We assume here that newborn cells have start with no activity, so we consider here only punctual proliferation at $x=0$. We assume that a fraction $\theta \in[0,1]$ of the energy is channelled to growth of the activity, and a fraction $(1-\theta)$ to the punctual proliferation. We also assume that for an individual of an activity value $x$ in the $k$ th subpopulation, $\theta g(P) x$ is the rate at which maintenance needs energy and $\theta g(P)(1-x)$ is what remains 
for growth.

Mathematically, we assume, according to the framework of the present paper, no dynamics for the energy resource and, therefore, the energy resource uptake rate $g$ will depend on the total population $P$, i.e. $g=g(P)$. In particular, $g(P)$ will be a smooth decreasing positive function satisfying $g(0)=1$ and tending to 0 when $|P|$ goes to $\infty$.

Thus we have the following sub-models for the growth and reproduction rates for each sub-population:

$$
v^{k}(P, x)=\theta v^{k} g(P)(1-x) \quad \text { and } \quad \beta^{k}(P, x)=(1-\theta) \beta^{k} g(P) x,
$$

where $v^{k}$ and $\beta^{k}$ are positive constants. In addition, we will assume the mortality rate $\mu^{k}$, for an individual in the $k$ th subpopulation, a function of $P$ only and positive. Based on the above modelling of cell interactions, the evolution system becomes as follows, for all $k \neq l=1,2$,

$$
\left\{\begin{array}{l}
\frac{\partial u^{k}(t, x)}{\partial t}+\theta v^{k} g(P(t)) \frac{\partial\left((1-x) u^{k}(t, x)\right)}{\partial x}+\mu^{k}(P(t)) u^{k}(t, x) \\
\quad=\frac{1}{1-\alpha^{k l}} \bar{u}^{k}\left(t, \frac{x}{1-\alpha^{k l}}\right) P^{l}(t)-u^{k}(t, x) P^{l}(t), \\
\theta v^{k} u^{k}(t, 0)=(1-\theta) \beta_{0}^{k} \int_{0}^{1} x u^{k}(t, x) \mathrm{d} x, \\
u^{k}(t, 0)=u_{k, 0}(x),
\end{array}\right.
$$

where

$$
\bar{u}:= \begin{cases}u & \text { if } x \in[0,1] \\ 0 & \text { otherwise }\end{cases}
$$

\subsection{Associate dynamical system}

We are interested in the evolution of the zeroth order moment $\left\{P^{k}(t)\right\}_{i=1}^{2}$ and the first order moments, related to the activity of each population:

$$
A^{k}(t)=\int_{0}^{1} x f^{k}(t)(x) \mathrm{d} x, \quad k=1,2 .
$$

Integrating (4.2) and multiplying (4.2) by $x$ and integrating also, and using (4.1) in both integrals, we obtain, after an integration by parts in the second one, the following ordinary differential equations system, for all $(k \neq l, k=1,2$ and $l=1,2)$ :

$$
\begin{cases}\dot{P^{k}}(t)=(1-\theta) \beta_{0}^{k} g(P) A^{k}(t)-\mu^{k}(P) P^{k}(t), & k=1,2, \\ \dot{A^{k}}(t)=\theta v^{k} g(P)\left(P^{k}(t)-A^{k}(t)\right)+\left(1-\alpha^{k l}\right) P^{l}(t) A^{k}(t)-\mu^{k}(P) A^{k}(t), & l=1,2, l \neq k .\end{cases}
$$

This system is supplemented by the initial conditions

$$
P^{k}(0)=\int_{0}^{1} f^{k}(0, x) \mathrm{d} x, \quad A^{k}(0)=\int_{0}^{1} x f^{k}(0, x) \mathrm{d} x .
$$

We now investigate the existence of steady states of (4.3). Let $\left(P_{1}^{*}, A_{1}^{*}, P_{2}^{*}, A_{2}^{*}\right)^{T}$ be a steady state of (4.3). It satisfies, for all $(i \neq j=1,2)$

$$
(1-\theta) \beta^{k} g\left(P^{*}\right) A_{k}^{*}=\mu^{k}\left(P^{*}\right) P_{k}^{*},
$$




$$
\theta v^{k} g\left(P^{*}\right)\left(P_{k}^{*}-A_{k}^{*}\right)=\left(1-\alpha^{k l}\right) P_{l}^{*} A_{k}^{*}-\mu^{k}\left(P^{*}\right) A_{k}^{*} .
$$

We find the following situations

1. Case 1: trivial steady state $(0,0,0,0)$.

2. Case 2: $\left(P_{1}^{*}, A_{1}^{*}, 0,0\right)^{T}$.

3. Case 3: $\left(0,0, P_{2}^{*}, A_{2}^{*}\right)^{T}$.

4. Case 4: Coexistence $\left(P_{1}^{*}, A_{1}^{*}, P_{2}^{*}, A_{2}^{*}\right)^{T}$.

In the last case of coexistence, the steady state is given by (4.5), we find

$$
\begin{aligned}
A_{1}^{*} & =\frac{\mu^{1}\left(P^{*}\right)}{(1-\theta) \beta^{1} g\left(P^{*}\right)} P_{1}^{*}, \\
A_{2}^{*} & =\frac{\mu^{2}\left(P^{*}\right)}{(1-\theta) \beta^{2} g\left(P^{*}\right)} P_{2}^{*}, \\
P_{1}^{*} & =\frac{\theta v^{2} g\left(P^{*}\right)\left((1-\theta) \beta_{0}^{2} g\left(P^{*}\right)-\mu^{2}\left(P^{*}\right)\right)}{\left(1-\alpha^{21}\right) \mu^{2}\left(P^{*}\right)}+\frac{\mu^{2}\left(P^{*}\right)}{1-\alpha^{21}}, \\
P_{2}^{*} & =\frac{\theta v^{1} g\left(P^{*}\right)\left((1-\theta) \beta_{0}^{1} g\left(P^{*}\right)-\mu^{1}\left(P^{*}\right)\right)}{\left(1-\alpha^{12}\right) \mu^{1}\left(P^{*}\right)}+\frac{\mu^{1}\left(P^{*}\right)}{1-\alpha^{12}} .
\end{aligned}
$$

\section{NumERICAL EXPERIMENTS}

\subsection{First case study}

\subsubsection{Experiment 1: Simulation with no proliferation by cell division}

Here we show the simulation results of the scheme (3.1). We compare the transfer process in the following three cases. In the first case, see (Fig. 2A top graphic), in the second case, we use only resistant cells (Fig. 2B middle graphic). In the third case see (Fig. $2 \mathrm{C}$ bottom graphic), initially we use a mixture between the type of cells, i.e. the initial data $u_{0}(x)$ is chosen as follows:

$$
u_{0}(x)=\left\{\begin{array}{lll}
u_{l} & \text { if } x \leq s_{1} & \text { sensitive cells } \\
0 & \text { if } s_{1} \leq x \leq s_{2}, & \\
u_{r} & \text { if } s_{2} \leq x \leq 1
\end{array} \quad\right. \text { resistant cells }
$$

The cells with small sizes of content of proteins are called sensitive cells to a therapy treatment and the cells with bigger sizes are called resistant cells. Here, $u_{l}$ and $u_{r}$ are given nonnegative constant data.

The results, in the case with no proliferation are shown in Figure 2, and in the case with proliferation in Figure 3. We use a uniform spatial grid over the interval $[0,1]$ with $\Delta x=0.1 / 8$ and 800 time iterations. We use two identical transfer rate functions $f_{1}(l)=f_{2}(l)=0.25$.

We can observe that for the both cases, the approximations tend to a Dirac function for large time iterations. We expect that the asymptotic behavior of the continuous solution converges to punctual Dirac function. All the cells tend to have the same quantity of proteins.

\subsubsection{Experiments 2 and 3: Effect of the transfer rate}

In this experiment, we compare the solution obtained with equal rate transfer $f_{1}(l)=f_{2}(l)$ and the solution obtained with inequal rate transfer $f_{1}(l) \neq f_{2}(l)$. We want to show that for short times the differences between the two solutions are quiet different. And one can use different transfer rates to capture and fit more precisely 

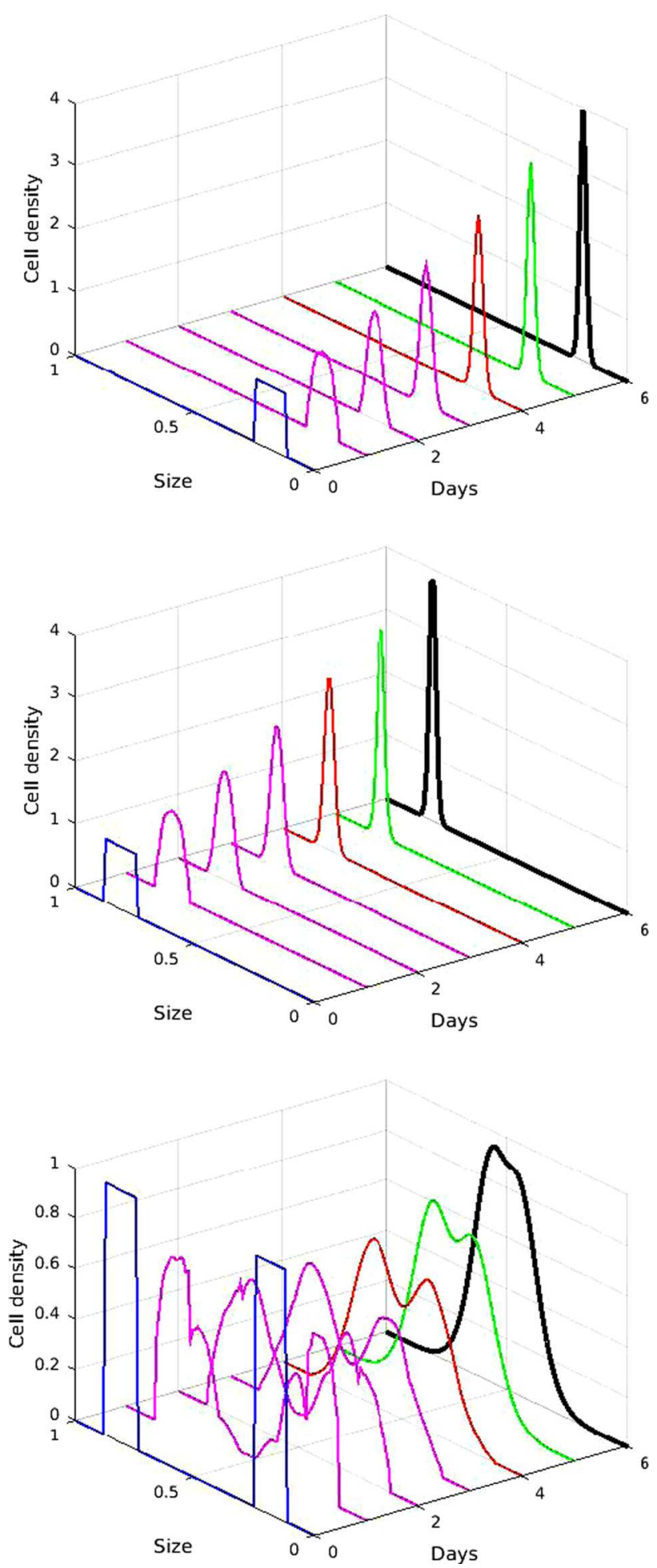

Figure 2. Experiment 1: (A) Top graphic: Sensitive cells, (B) Middle graphic: Resistant cells, and $(\mathrm{C})$ bottom graphic: Mixture sensitive and resistance cells. We use equal transfer rates $f_{1}=f_{2}=0.2$. 


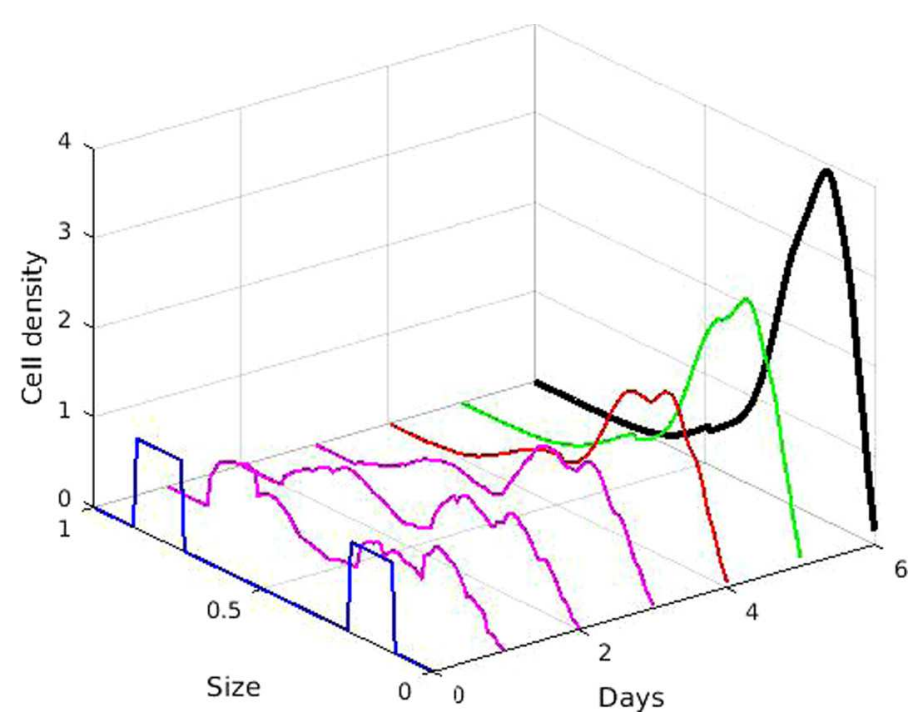

Figure 3. Proliferation with equal cell division; unequal transfer rates $f_{1}=0.5$ and $f_{2}=0.45$.
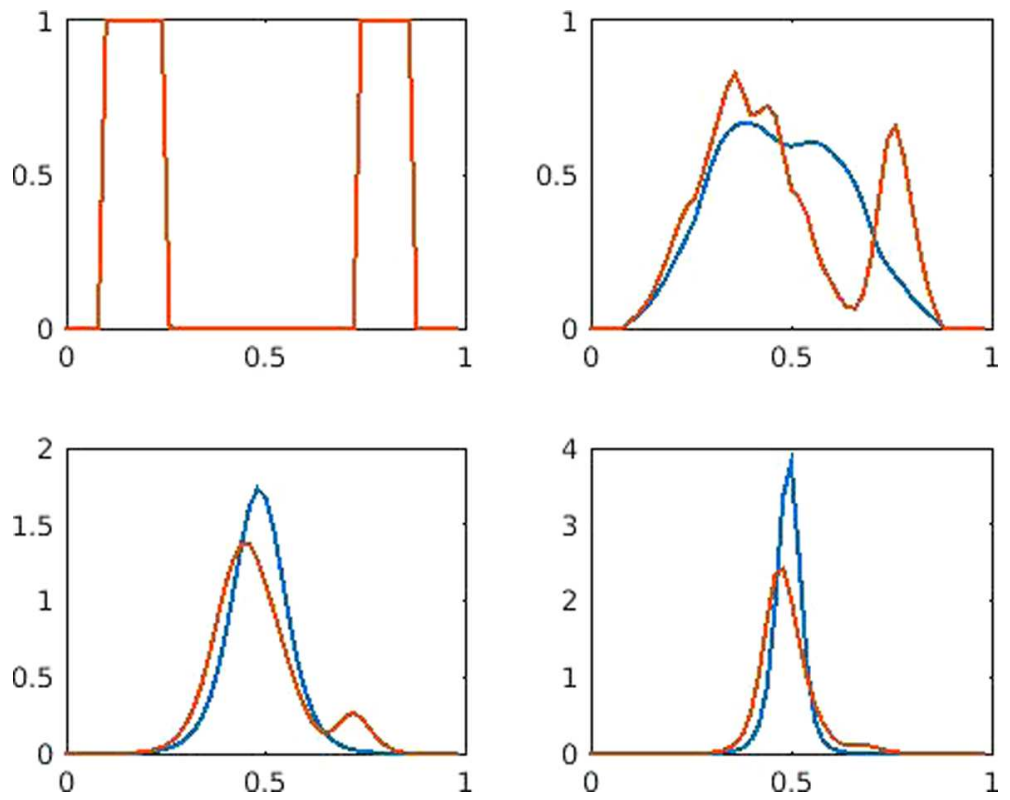

FIGURE 4. Experiment 2: Effect of the transfer rate: we compare the solution with equal rate $f_{1}=f_{2}=0.25$, and the solution with inequal transfer rates $f_{1}=0.25$ and $f_{2}=0.05$. (A) Top left: at time $t=0$ initial data. (B) Top right: after 2 days. (C) Bottom left: after 4 days. (D) Bottom right: after 6 days.

experimental data. However the large time behavior is quiet similar. The solutions converge to a Dirac function. This means that after long time, all the cells reach to have the same content of shred proteins (Figs. 4 and 5). 

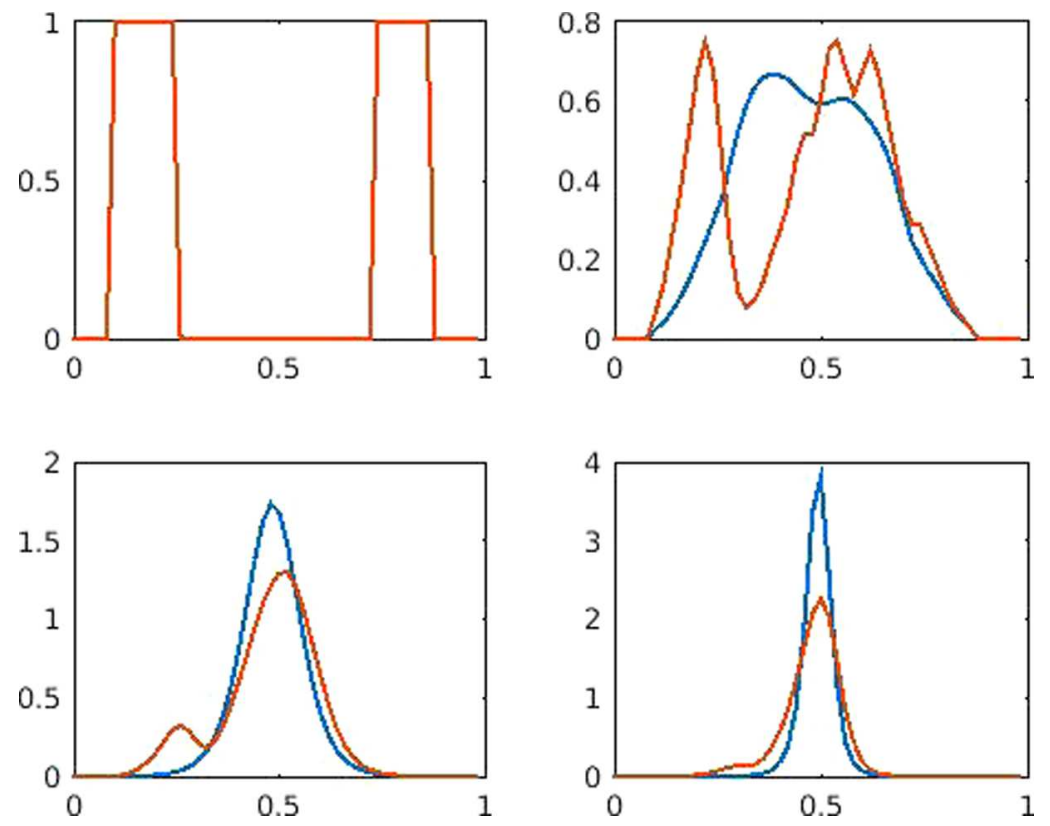

FiguRE 5. Experiment 3: Effect of the transfer rate: we compare the solution with $f_{1}=0.25$ and $f_{2}=0.25$, and the solution with inequal transfer rates $f_{1}=0.05$ and $f_{2}=0.25$. (A) Top left: at time $t=0$ initial data. (B) Top right: after 2 days. (C) Bottom left: after 4 days. (D) Bottom right: after 6 days.

\subsubsection{Experiment 3: Simulation with distributed proliferation}

In this situation, the system (5.2) becomes

$$
\begin{cases}\frac{\partial u(t, x)}{\partial t}=Q(u(t, \cdot), u(t, \cdot))(x)+4 b(2 x) u(t, 2 x)-b(x) u(t, x) & \text { for } x \in \mathbb{R}, \\ u(0, \cdot)=u_{0} \in L_{+}^{1}(\mathbb{R}) . & \end{cases}
$$

The terms containing $b(x)$ and $b(2 x)$, describe the division of cells of size $2 x$ into two cells of size $x$. The total number of new cells created per time is then

$$
\int_{0}^{1} b(x) u(t, x) \mathrm{d} x
$$

the function $b(\cdot)$ is the rate of division per unit of time. One can check that no extra content of protein is added by cell division it means that

$$
\int_{0}^{1} 4 x b(2 x) u(t, 2 x) \mathrm{d} x=\int_{0}^{1} x b(x) u(t, x) \mathrm{d} x .
$$

\subsection{Second case study: Immune-tumor}

This subsection is devoted to simulations of the proposed model of immune and tumor competition. We deal with the simulation of the dynamics of the two population models of the model (4.2). To ovoid complexity for well understanding the present paper and for more simplicity to understand the qualitative behaviors and it biological interpretation. We assume that the growth rates, $v^{k}(\mathbf{P}, x)$, the mortality rates $\mu^{k}(\mathbf{P})$ used in the 
TABLE 1. Different values of the model parameters.

\begin{tabular}{cccc}
\hline Parameter & Experiment 4 & Experiment 5 & \\
\hline$v^{1}$ & 1 & 1 & Growth rate \\
$v^{2}$ & 1 & 1 & \\
$\beta^{1}$ & 0.1 & 0.1 & Birth rate \\
$\beta^{2}$ & 0.1 & 0.1 & \\
$\mu^{1}$ & 0.01 & 0.01 & Mortality rate \\
$\mu^{2}$ & 0.01 & 0.01 & \\
$\theta$ & 0.5 & 0.5 & See $(4.1)$ \\
$\alpha_{12}$ & $0.1,0.25,0.9$ & 0.1 & Depletion rate \\
$\alpha_{21}$ & 0.25 & $0.1,0.25,0.9$ & \\
\hline
\end{tabular}
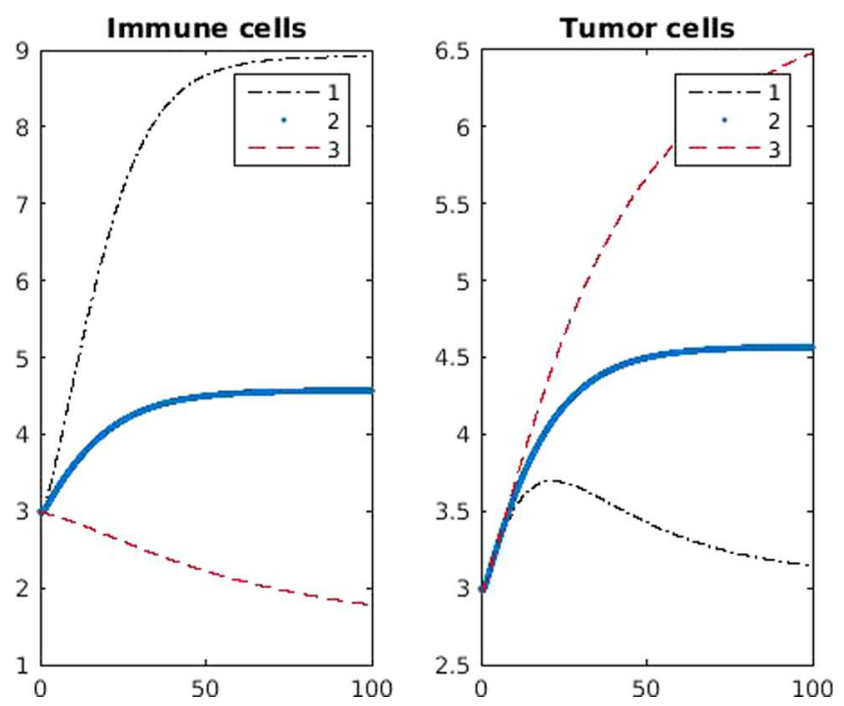

Figure 6. Effect of depletion rate: we use $\alpha_{21}=0.25$ and respectively: (1) $\alpha_{21}>\alpha_{12}$ with $\left(\alpha_{12}=0.05\right),(2) \alpha_{21}=\alpha_{12}=0.25$ and (3) $\alpha_{21}<\alpha_{12}$ with $\left(\alpha_{12}=0.75\right)$.

model are constant functions. More complicated examples of these functions can be found in [2]. The different values of the parameters used in these simulations are given in Table 1.

With this simplification the steady state, in the case of coexistence of the cells, takes the following expression: from (4.7), we find

$$
\begin{aligned}
& A_{1}^{*}=\frac{\mu^{1}}{(1-\theta) \beta^{1}} P_{1}^{*}, \\
& A_{2}^{*}=\frac{\mu^{2}}{(1-\theta) \beta^{2}} P_{2}^{*}, \\
& P_{1}^{*}=\frac{\theta v^{2}\left((1-\theta) \beta_{0}^{2}-\mu^{2}\right)}{\left(1-\alpha^{21}\right) \mu^{2}}+\frac{\mu^{2}}{1-\alpha^{21}}, \\
& P_{2}^{*}=\frac{\theta v^{1}\left((1-\theta) \beta_{0}^{1}-\mu^{1}\right)}{\left(1-\alpha^{12}\right) \mu^{1}}+\frac{\mu^{1}}{1-\alpha^{12}} .
\end{aligned}
$$


$\mathbf{P 2}$ versus $\mathbf{P 1}$

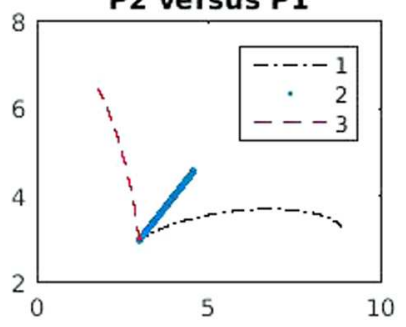

A2 versus $\mathbf{P 2}$

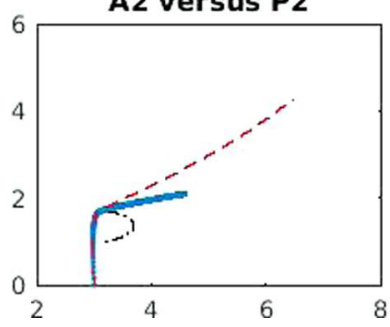

Al versus S P1

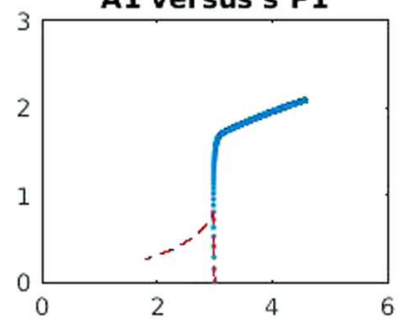

A2 versus $\mathrm{A1}$

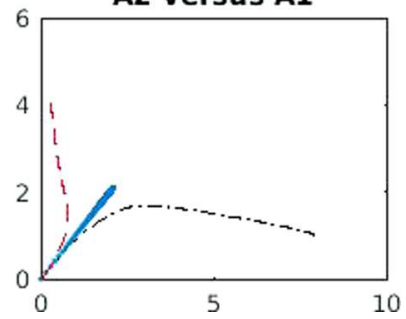

Figure 7. Different phase portrait of the solution of the same experiment as above. We use $\alpha_{21}=0.25$ and respectively: (1) $\alpha_{21}>\alpha_{12}$ with $\left(\alpha_{12}=0.05\right),(2) \alpha_{21}=\alpha_{12}=0.25$ and (3) $\alpha_{21}<\alpha_{12}$ with $\left(\alpha_{12}=0.75\right)$.

In these simulations, we show the evolution in time of the population of immune cells (left graphic Fig. 6) and of the population of cancer cells (right graphic Fig. 6). On each picture, we can observe the effect of the depletion factors $\alpha_{1,2}$ and $\alpha_{1,2}$.

1. For $\alpha_{12}<\alpha_{21}$, correspond to the case where immune cells to inhibit cancer cells. We observe that the final output, is the reduction of the state of abnormal cells until their complete depletion and a final survival of immune cells. The population of immune cells increases due to its increasing activity, to reach its asymptotic state. The population of cancer cells go to extinction then are destroyed, see Figure 6(2).

2. For $\alpha_{12}=\alpha_{21}$, the two populations of immune cells and cancer cells survive and reach a positive steady state, seen in Figure 6(2).

3. If $\sigma_{12}<\sigma_{21}$, the ability of abnormal cells to inhibit immune cells is greater than the ability of immune cells to reduce the state of abnormal cells. We observe that the final output is the reduction of the population of immune cells until their complete depletion and a final survival of the cancer cells. The population of cancer cells increases due to its increasing activity, to reach its asymptotic state. The population of immune cells is reduced, see Figure 6(2).

\section{CONCLUSiOn}

In this paper we have presented a novel class of population dynamic inspired from kinetic theory of active particles.

In the first case study, a discrete model was formulated to describe some transfer rules in population constituted by sensitive cells and resistant cells. The resistance to therapies is characterized by a large quantity of proteins located along the boundary membrane of cells acting as a barrier to cytotoxic substances. The population of cell is then structured by a discrete variable corresponding to size of transferable material. We have derived a discrete model and proved the convergence from a discrete to a continuous model which is one-dimensional Boltzmann-type equation of active particles with avoiding the spatial mechanics aspect. In the second part of the paper, we have shown a mathematical model of a two species immune cells and tumor cells. We have provided a qualitative analysis of the large time behavior of the competition between progressing (tumor) cells and immune cells. We have given numerical solutions showing the convergence to different steady 
states depending on the values of the depletion rates of each type of cells. In conclusion this global formalism described in the first section provides a model to study the cell-to-cell direct interactions with a Boltzmann-like approach avoiding the complexity of the spatial structure Boltzmann equation which deals with mechanical structure of the collision between cells (Fig. 7).

\section{Appendix A. NumericAl SCHEME}

For a self-contency of the paper, we add in this appendix the useful properties of the numerical scheme solving the system (4.1). Selecting a mesh size $\Delta x>0$, a time step $\Delta t>0$ and a final time $T>0$ we show how to obtain the approximate solution in the interval $[0, T] \times[0,1]$. We use the framework of finite volume method and we define a generalized upwind scheme. We set for any $i=1, \ldots, I, x_{1 / 2}=0$ and $x_{i+1 / 2}=x_{1 / 2}+i \Delta r$ with $I$ being the integer part of $1 / \Delta x$. We define the first mesh cell $\left.M_{1}^{\Delta}=\right] 0, x_{1+1 / 2}$ [ with the middle point $x_{I}=$ $(I-1 / 2) \Delta x$, and the last mesh cell $\left.M_{I}^{\Delta}=\right] x_{I-1 / 2}, 1\left[\right.$ with the middle point $x_{1}=\Delta x / 2$. We define the meshes $\left.M_{i}^{\Delta}=\right] x_{i-1 / 2}, x_{i+1 / 2}\left[\right.$ with the middle point $x_{i}=x_{i-1 / 2}+\Delta x / 2$ for any $i \in \mathbb{N}^{*}$. In this way the interval $[0,1]$ is discretized with the cells $M_{i}^{\Delta}$. The approximate solution $u^{k, \Delta}$ (for each family $k, k=1, \ldots, N_{f}$ ), generated by the scheme, at time $t_{n}$ is denoted $u^{k, n}(x)=u^{k, \Delta}\left(t_{n}, x\right)$ and $u^{k, n}(\cdot)$ is built in such a way to lie in $V^{n, \Delta}$, with the following set of piecewise constant functions:

$$
V^{n, \Delta}=\left\{v^{n}(\cdot), \text { such that } v^{n}(x)=v_{i}^{n} \text { if } x \in M_{i}^{\Delta}\right\} .
$$

The numerical scheme is really a transport projection method, that is a combination of $S^{\Delta}(t)$-operator projection operator on the discrete space. It corresponds to the following formulation:

$$
u^{k, n+1, \Delta}=P^{\Delta} S^{\Delta}(\Delta t) u^{k, n, \Delta},
$$

and more explicitly the scheme takes the following form:

$$
\left\{\begin{aligned}
u_{i}^{k, n+1}= & u_{i}^{k, n}-\frac{\Delta t}{\Delta x}\left(v_{i+1 / 2}^{k, n} u_{i}^{k, n}-v_{i-1 / 2}^{k, n} u_{i-1}^{k, n}\right)+\Delta t \sum_{l=1}^{N_{f}} Q_{i}^{k l, n} \\
& -\Delta t \mu_{i}^{k, n} u_{i}^{k, n}+\Delta t \sum_{j} B_{i, j}^{k, n} u_{j}^{n} \Delta x, \\
v_{1 / 2}^{k, n} u_{0}^{k, n}= & \sum_{i=1}^{I_{L}} \beta_{i}^{k, n} u_{i}^{k, n} \Delta x .
\end{aligned}\right.
$$

This scheme works under the CFL condition (A.3), i.e.

$$
\max _{k}\left(\sup _{x} v^{k, n}(x)\right) \frac{\Delta t}{\Delta x} \leq 1
$$

We give some estimates satisfied by the scheme. We have the following lemma concerning the positiveness satisfied by the solutions of the scheme.

Lemma A.1. Starting with $u_{i}^{k, p} \geq 0$, for all $i, k$ and $v^{k, p} \geq 0$. Choosing $\Delta t$ so small that

$$
\frac{\Delta t}{\Delta x} \leq \frac{1}{\sup _{i \leq I_{L}}\left(v_{i+1 / 2}^{k, p}+\Delta x\left(\mu_{i}^{k, p}+\eta_{\infty} \sum_{l=1}^{n}\left|u^{l, p}\right|_{1, \Delta}\right)\right)}
$$


for all $p \leq n$, we obtain $u_{i}^{k, n+1}$ positive. Moreover, let $u_{0}^{k} \in L^{1}(] 0,1[) \cap L^{\infty}(] 0,1[)$, the discrete $L^{1}$-norm satisfies

$$
\left|u^{k, n}\right|_{1, \Delta} \leq e^{\max _{k}\left(\left|\beta^{k}\right|_{\infty}+\left|\sum_{j} B_{i, j}^{k, n}\right|_{\infty}\right)} T_{\left|u^{k, 0}\right|_{1, \Delta},}
$$

the discrete $L^{\infty}$-norm satisfies

$$
\left|u^{k, n}\right|_{\infty, \Delta} \leq e^{\left(\max _{k}\left|v^{k, n}\right|_{L i p}+\eta_{\infty} N_{f} \max _{k}\left|u^{k, n}\right|_{1, \Delta}\right) T}\left|u^{k, 0}\right|_{\infty, \Delta}+C(T)
$$

with $C(T)$ being constant independent of $n$.

The total variation bound plays a crucial role in establishing the subsequential convergence of the difference approximation scheme. In the next lemma we give the boundness of the total variation of the approximate solution.

Lemma A.2. Let $u_{0}^{k} \in\left(L^{1} \cap L^{\infty}\right)(] 0,1[)$ with the total variation be bounded locally in $] 0,1[$ and under the CFL condition (A.3), then we have

$$
T V\left(u^{k, n+1}\right) \leq e^{K T}\left(T V\left(u^{k, 0}\right)+M\right)
$$

with $M$ and $K$ being constants independent of $n$. The total variation in time satisfies

$$
\sum_{i=1}^{I_{L}}\left|u_{i}^{k, n+1}-u_{i}^{k, n}\right| \leq T V\left(u^{k, n}\right)+r C(T)
$$

which implies the $L^{1}$ Lipschitz continuity in time.

Proof. A quiet similar proof can be found in [2].

Now we deal with the existence of global weak solution using Helly compactness argument, based on the compact canonical imbedding from $W^{1,1}(] 0,1[)$ into $L^{1}(] 0,1[)$. We first prove the existence of a limit $u^{k}$ to $u^{k, \Delta}$ as the meshsize $\Delta x$ goes to zero. Then we prove that this limit is a weak solution of the problem (4.1).

Theorem A.3. Let $u_{0}^{k} \in\left(L^{1} \cap L^{\infty}\right)(] 0,1[)$ with total variation bounded locally in $] 0,1\left[\right.$, $u_{0}^{k} \geq 0$, then as the meshsize $\Delta x$ tends to zero, there is a subsequence of $\left(u^{k, \Delta}\right)_{\Delta x>0}$, the family of approximate solution, converging in $L_{l o c}^{1}([0, T] \times] 0,1[)$ to a function $u^{k} \in L_{l o c}^{1}([0, T] \times] 0,1[)$.

Proof. A quiet similar proof can be found in [2].

\section{Appendix B. Proof of theorem 3.1}

\section{B.1 Existence of a limit $u$}

We first prove the existence of a limit $u$ to $u^{\Delta}$ as the mesh size $\Delta x$ goes to zero. Then we prove that this limit is a solution of the continuous problem (5.2).

The proof of existence of the limit $u(t, x)$ is based on the compact canonical embedding from $W^{1,1}(] 0,1[)$ into $L^{1}(] 0,1[)$. Let $I\left(u^{\Delta}\right)$, defined on $\left.[0, T] \times\right] 0,1\left[\right.$, be the interpolate of degree one of $u^{\Delta}$ at the vertices of each 
rectangle $\left[x_{i}, x_{i+1}\right] \times\left[t_{n}, t_{n+1}\right]$; where it is given by

$$
\begin{aligned}
I\left(u^{\Delta}\right)(x, t)= & u_{i}^{n}+\left(u_{i+1}^{n}-u_{i}^{n}\right) \frac{x-i \Delta x}{\Delta x}+\left(u_{i}^{n+1}-u_{i}^{n}\right) \frac{t-n r \Delta x}{r \Delta x} \\
& +\left(u_{i+1}^{n+1}-u_{i}^{n+1}-u_{i+1}^{n}+u_{i}^{n}\right) \frac{(x-i \Delta x)(t-n r \Delta x)}{r(\Delta x)^{2}},
\end{aligned}
$$

$I\left(u^{\Delta}\right)$ is continuous with

$$
\left|I\left(u^{\Delta}\right)\right|_{L^{\infty}([0, T] \times] 0,1[)}=\left|u^{\Delta}\right|_{L^{\infty}([0, T] \times] 0,1[)}=\sup _{n, i}\left|u_{i}^{n}\right|,
$$

and differentiable inside each rectangle. Thus we obtain

$$
\iint\left|\frac{\partial I\left(u^{\Delta}\right)}{\partial t}\right| \mathrm{d} x \mathrm{~d} t \leq \sum_{n=0}^{N} \sum_{i \leq I_{L}}\left|u_{i}^{n+1}-u_{i}^{n}\right| \Delta x .
$$

In the same way

$$
\iint\left|\frac{\partial I\left(u^{\Delta}\right)}{\partial x}\right| \mathrm{d} x \mathrm{~d} t=\sum_{n=0}^{N} \sum_{i \leq I_{L}}\left|u_{i+1}^{n}-u_{i}^{n}\right| r \Delta x
$$

On the other hand one can check that the numerical scheme (3.1) satisfies the following a priori estimates

$$
\begin{aligned}
& \sup _{i}\left|u_{i}^{n+1}\right| \leq\left(1+C_{1} \Delta t\right) \sup _{i}\left|u_{i}^{n}\right|, \\
& \sum_{i=1}^{I_{L}}\left|u_{i+1}^{n+1}-u_{i}^{n+1}\right| \leq\left(1+C_{2} \Delta t\right) \sum_{i=1}^{I_{L}}\left|u_{i+1}^{n}-u_{i}^{n}\right|, \\
& \sum_{i=1}^{I_{L}}\left|u_{i}^{n+1}-u_{i}^{n}\right| \leq \sum_{i=1}^{I_{L}}\left|u_{i+1}^{n}-u_{i}^{n}\right|,
\end{aligned}
$$

where $C_{1}$ and $C_{2}$ are two constant independents of $n$.

Let $u_{0} \in L^{\infty}(] 0,1[)\left(i . e . \sup _{i}\left|u_{i}^{0}\right| \leq C\right)$ and with the total variation $T V\left(u_{0}(x)\right)=\sum_{i=1}^{I_{L}}\left|u_{0}\left(x_{i+1}\right)-u_{0}\left(x_{i}\right)\right|$ be bounded, we have, by applying the discrete Gronwall lemma to the estimates (B.5) and using successively (B.2)-(B.4), it follows that $u^{\Delta}$ is bounded and contains a subsequence $u^{\Delta_{p}}$ weakly star convergent to a limit $u \in L^{\infty}([0, T] \times] 0,1[)$ bounded by $\left|u_{0}\right|_{L}^{\infty}(] 0,1[)$. It follows also that:

$$
\left|I\left(u^{\Delta}\right)\right|_{L^{\infty}([0, T] \times] 0,1[)}+\left|\frac{\partial I\left(u^{\Delta}\right)}{\partial x}\right|_{L^{1}([0, T] \times] 0,1[)}+\left|\frac{\partial I\left(u^{\Delta}\right)}{\partial t}\right|_{L^{1}([0, T] \times] 0,1[)} \leq M .
$$

Therefore from $\left\{I(u)^{\Delta}\right\}$ associated to $\left\{u^{\Delta}\right\}$, we extract a subsequence convergent to $\left\{I(u)^{\Delta_{p}}\right\}$ in $L_{l o c}^{1}(] 0, T[\times] 0,1[)$. Then we verify that $\left\{I(u)^{\Delta_{p}}-u^{\Delta_{p}}\right\}$ tends to zero in $L^{1}$, for all bounded open sets ] $0, T[\times] 0,1\left[\right.$. Since the associate subsequence $u^{\Delta}$ weakly star converges to a function $u \in L^{\infty}(] 0, T\left[\times \Omega_{L}\right)$, and since on the other hand $\left\{I\left(u^{\Delta_{p}}\right)\right\}$ is convergent in $L_{l o c}^{1}(] 0, T[\times] 0,1[)$, we have

$$
u^{\Delta} \text { converges to } u \text { in } L_{l o c}^{1}(] 0, T[\times] 0,1[) .
$$


This end the proof of the existence of a limit.

\section{B.2 Convergence of $Q^{\Delta}\left(u^{\Delta}, u^{\Delta}\right)$ to $Q(u, u)$}

Now we prove the following lemma:

Lemma B.1. $Q^{\Delta}\left(u^{\Delta}, u^{\Delta}\right)$ converges to the continuous transfer operator $Q(u, u), u$ being the limit function of $u^{\Delta}$ as $\Delta x$ goes to zero.

Proof. Let us write

$$
\left|Q^{, \Delta}\left(u^{\Delta}, u^{\Delta}\right)-Q(u, u)\right|_{1} \leq\left|Q^{\Delta}\left(u^{\Delta}, u^{\Delta}\right)-Q\left(u^{\Delta}, u\right)\right|_{1}+\left|Q\left(u^{\Delta}, u\right)-Q(u, u)\right|_{1}
$$

Since the transfer operator $\mathrm{Q}$ is locally Lipschitz and $u^{\Delta}$ converges to $u$ the second term of the right hand side of the above inequality $\int_{0}^{1}\left|Q^{\Delta}\left(u^{\Delta}, u\right)(x)-Q(u, u)(x)\right| \mathrm{d} x \longrightarrow 0$ as the mesh size $\Delta x$ goes to zero.

Let now calculate $Q\left(u^{\Delta}, u^{\Delta}\right)(x)$.

$$
\begin{aligned}
Q\left(u^{\Delta}, u^{\Delta}\right)(x)= & \sum_{j} \int_{x_{j}}^{x_{j+1}} \pi_{1}(x) u^{\Delta}\left(x_{i}+f_{1}(x) p\right) u^{\Delta}\left(x_{i}-\left(1-f_{1}(x)\right) p\right) \mathrm{d} x \\
& +\sum_{j} \int_{x_{j}}^{x_{j+1}} \pi_{2}(x) u^{\Delta}\left(x_{i}+f_{2}(x) p\right) u^{\Delta}\left(x_{i}-\left(1-f_{2}(x)\right) p\right) \mathrm{d} x .
\end{aligned}
$$

since $u^{\Delta}$ is piecewise constant function we have

$$
\begin{aligned}
Q\left(u^{\Delta}, u^{\Delta}\right)(x)= & \sum_{j} \int_{x_{j}}^{x_{j+1}} \pi_{1}(x) \mathrm{d} x u_{i+f_{1}\left(p_{j}\right) j}^{n} u_{i-\left(1-f_{1}\left(p_{j}\right)\right) j}^{n} \\
& +\sum_{j} \int_{x_{j}}^{x_{j+1}} \pi_{2}\left(p_{j}\right) \mathrm{d} x \bar{u}_{i+f_{2}\left(p_{j}\right) j}^{n} \bar{u}_{i-\left(1-f_{2}(x)\right) j}^{n}
\end{aligned}
$$

but using the mean values of $\pi_{1}$ and $\pi_{2}$

$$
\pi_{1}\left(l_{j}\right)=\frac{\int_{x_{j}}^{x_{j+1}} \pi_{1}(x) \mathrm{d} x}{\Delta x} \text { and } \pi_{2}\left(l_{j}\right)=\frac{\int_{x_{j}}^{x_{j+1}} \pi_{2}(x) \mathrm{d} x}{\Delta x}
$$

we conclude that $Q\left(u^{\Delta}, u^{\Delta}\right)-Q^{\Delta}\left(u^{\Delta}, u^{\Delta}\right)=0$. Then $\int_{0}^{1}\left|Q^{\Delta}\left(u^{\Delta}, u^{\Delta}\right)(x)-Q\left(u^{\Delta}, u\right)(x)\right| \mathrm{d} x \longrightarrow 0$ as the mesh size $\Delta x$ goes to zero. This completes the proof of the lemma.

\section{B.3 Weak solution}

Now we consider the consistency of the scheme, it means that this limit $u$ of the discrete solutions $u^{\Delta}$ is a weak solution of the continuous problem.

For all smooth $\phi \in C^{1}([0, T] \times] 0,1[)$ with compact support in $[0, T[\times] 0,1[$, we define

$$
\forall(t, x) \in\left[x_{i-1}, x_{i}\left[\times\left[t_{n}, t_{n+1}\left[, \quad \phi^{\Delta}(t, x)=\phi_{i}^{n}=\frac{1}{\Delta t \Delta x} \int_{t_{n}}^{t_{n+1}} \int_{x_{i-1}}^{x_{i}} \phi(t, x) \mathrm{d} t \mathrm{~d} x .\right.\right.\right.\right.
$$


Multiplying the scheme (3.1) by $\Delta x \phi_{i}^{n}$ we get,

$$
\sum_{i, n}\left(u_{i}^{n+1}-u_{i}^{n}\right) \phi_{i}^{n} \Delta x-\Delta t \sum_{i, n}\left(Q_{i}^{n} \phi_{i}^{n} \Delta x+\sum_{j} B_{i, j}^{n} u_{j}^{n} \Delta x\right) \phi_{i}^{n} \Delta x=0,
$$

then summing by part we get

$$
\sum_{i, n}\left(u_{i}^{n+1}\left(\phi_{i}^{n}-\phi_{i}^{n+1}\right)\right) \Delta x-\Delta t\left(\sum_{i, n} Q_{i}^{n} \phi_{i}^{n} \Delta x+\sum_{j} B_{i, j}^{n} u_{j}^{n} \Delta x\right) \phi_{i}^{n} \Delta x-\sum_{i} u_{i}^{0} \phi_{i}^{0} \Delta x=0,
$$

which is equivalent to

$$
\begin{aligned}
& \int_{0}^{T} \int_{] 0,1[} u^{\Delta}(t, x) \frac{\phi^{\Delta}(t+\Delta t, x)-\phi^{\Delta}(t, x)}{\Delta t} \mathrm{~d} x \mathrm{~d} t+\int_{0}^{T} \int_{] 0,1[} Q^{\Delta}\left(u^{\Delta}, u^{\Delta}\right)(t, x) \phi^{\Delta}(t, x) \mathrm{d} x \mathrm{~d} t \\
& \int_{] 0,1[} \int_{0}^{1} B^{\Delta}\left(P^{n}, x, \xi\right) u^{\Delta}(t, \xi) \mathrm{d} \xi \mathrm{d} x+\int_{] 0,1[} u^{\Delta}(0, x) \phi^{\Delta}(0, x) \mathrm{d} x=0,
\end{aligned}
$$

we pass to the limit $\Delta x \rightarrow 0$, we obtain

$$
\begin{aligned}
& \int_{0}^{T} \int_{] 0,1[} u(t, x) \frac{\partial \phi}{\partial t}(t, x) \mathrm{d} x \mathrm{~d} t+\int_{0}^{T} \int_{\Omega_{L}} Q(u, u) \phi(t, x) \mathrm{d} x \mathrm{~d} t \\
& \quad+\int_{] 0,1[} \int_{0}^{1} B(P(t), x, \xi) u(t, \xi) \mathrm{d} \xi \mathrm{d} x+\int_{] 0,1[} u(0, x) \phi(0, x) \mathrm{d} x=0,
\end{aligned}
$$

which means that the limit $\mathrm{u}$ obtained using the discrete scheme is a weak solution of the problem (5.2) with the initial data $u_{0}(x)$.

\section{REFERENCES}

[1] S.V. Ambudkar, Z.E. Sauna, M.M. Gottesman and G. Szakacs, A novel way to spread drug resistance in tumor cells: functional inter cellular transfer of P-glycoprotein (ABCB1). Trends Pharmacol. Sci. 26 (2005) 385-387.

[2] B. Aylaj and A. Noussair, Convergence of numerical schemes to a nonlinear kinetic model of population dynamics with nonlocal boundary conditions. SIAM J. Numer. Anal. Soc. Ind. Appl. Math. 48 (2010) 1707-1732.

[3] N. Bellomo, Modeling Complex Living Systems: A Kinetic Theory and Stochastic Game Approach. Springer Science and Business Media, Basel (2008).

[4] N. Bellomo, N. Li and P. Maini, On the foundations of cancer modelling selected topics, speculations, and perspectives. Math. Models Meth. Appl. Sci. 18 (2008) 593-646.

[5] A. Bellouquid and M. Delitala, Mathematical Modeling of Complex Biological Systems: A Kinetic Theory Approach. Birkhäuser, Boston, Basel, Berlin (2006).

[6] B. Dale, G.P. McNerney, D.L. Thompson, W. Hübner, T. Huser, B.K. Chen, Visualizing cell-to-cell transfer of HIV using fluorescent clones of HIV and live confocal microscopy. J. Vis. Exp. 44 (2010) 2061-2061.

[7] D.M. Davis, T. Igakura, F.E. McCann, L.M. Carlin, K. Andersson, B. Vanherberghen et al., The protean immune cell synapse: a supramolecular structure with many functions. Semin. Immunol. 15 (2003) 317-324.

[8] M.E. Dudley and S.A. Rosenberg, Adoptive-cell-transfer therapy for the treatment of patients with cancer. Nat. Rev. Cancer 3 (2002) 666-675.

[9] M.E. Dudley, J.R. Wunderlich, J.C. Yang, R.M. Sherry, S.L. Topalian, N.P. Restifo et al., Adoptive cell transfer therapy following non-myeloablative but lymphodepleting chemotherapy for the treatment of patients with refractory metastatic melanoma. J. Clin. Oncol. 23 (2005) 2346-2357.

[10] C. Hansen, E. Angot, A.L. Bergstrom, J.A. Steiner, L. Pieri, G. Paul et al., $\alpha$-Synuclein propagates from mouse brain to grafted dopaminergic neurons and seeds aggregation in cultured human cells. J. Clin. Invest. 121 (2011) 715-725. 
[11] A. Levchenko, B.M. Mehta, X. Niu, G. Kang, L. Villafania, D. Way et al., Intercellular transfer of P-glycoprotein mediates acquired multidrug resistance in tumor cells. Proc. Natl. Acad. Sci. USA 102 (2005) 1933-1938.

[12] R.H. Martin, Jr., Nonlinear Operators and Differential Equations in Banach Spaces. Wiley, New York (1976).

[13] A.S. Novozhilov, G.P. Karev and E. Koonin, Mathematical modeling of evolution of horizontally transferred genes. Mol. Biol. Evol. 22 (2005) 1721-1732.

[14] Y. Suhail, Kshitiz, J. Lee, M. Walker, M.D. Brennan, J.S. Bader et al., Modeling intercellular transfer of biomolecules through tunneling nanotubes. Bull. Math. Biol. 75 (2013) 1400-1416.

[15] C. Villani, A review of mathematical topics in collisional kinetic theory, in Vol. 1 of Handbook of Mathematical Fluid Dynamics, edited by S. Friedlander and D. Serre. North-Holland, Amsterdam (2002) 71-305. 